\title{
Comprehensive proteomic datasets for studying adipocyte-macrophage cell-cell communication
}

\author{
Anja Freiwald, Christopher Weidner, Annabell Witzke, Sheng-Yu Huang, David Meierhofer \\ and Sascha Sauer
}

Otto Warburg Laboratory, Max Planck Institute for Molecular Genetics, Berlin, Germany

Cellular communication is a fundamental process in biology. The interaction of adipocytes with macrophages is a key event in the development of common diseases such as type 2 diabetes. We applied an established bilayer cell coculture system and comprehensive MS detection to analyse on a proteome-wide scale the paracrine interaction of murine adipocytes and macrophages. Altogether, we identified 4486 proteins with at least two unique peptides, of which 2392 proteins were informative for 3T3-L1 adipocytes and 2957 proteins for RAW 264.7 macrophages. Further, we observed over 12000 phosphorylation sites, of which we could assign 3200 informative phosphopeptides with a single phosphosite for adipocytes and 4514 for macrophages. Using protein set enrichment and phosphosite analyses, we deciphered regulatory protein pathways involved in cellular stress and inflammation, which can contribute to metabolic impairment of cells including insulin resistance and other disorders. The generated datasets provide a holistic, molecular pathway-centric view on the interplay of adipocytes and macrophages in disease processes and a resource for further studies.

\section{Keywords:}

Cell biology / Cell signalling / Homeotasis / Metabolic diseases / SILAC / Transwell system

Additional supporting information may be found in the online version of this article at the publisher's web-site

Metabolic diseases such as obesity and type 2 diabetes are a major burden on human health. These multifactorial disorders imply a number of deregulated organs and interconnected cellular pathways. Importantly, in obese subjects, chronic low-grade inflammation of adipose tissue containing infiltrated macrophages contributes to increasing insulin resistance, a hallmark of type 2 diabetes [1,2]. A number of mechanistic studies have shown that elevated levels of free fatty acids activate macrophages by interacting via fetuin-A with the Toll-like receptor 4 [3]. This activation leads to secretion of cytokines of macrophages, which interfere with adipocytes resulting in impaired insulin sensitivity including decreased insulin-stimulated glucose uptake in adipocytes [4-6]. Consequently, inhibition of inflammatory processes in adipose tissue was efficient to counteract insulin resis-

Correspondence: Dr. Sascha Sauer, Otto Warburg Laboratory, Max Planck Institute for Molecular Genetics, Ihnestrasse 63-73, 14195 Berlin, Germany

E-mail: sauer@molgen.mpg.de

Abbreviation: PSEA, protein set enrichment analysis tance [7], but potentially other, for example microbial factors also played a role.

In principle, shotgun or targeted proteomics based on quantitative MS is a powerful methodology to systematically explore the underlying pathways of cell homeostasis and pathophysiology. However, conventional large-scale proteomics studies investigate only one cell line at the time [8]. The proteome-wide cellular changes due to the influence of circumjacent cells are commonly analyzed indirectly, for example by treating cells with other cells' supernatant or using a cocktail of signaling molecules [9].

Coculturing of two cell lines using a porous membrane creates a microenvironment for cell-cell communication and exchange of media and signaling molecules. This transwell system allows the harvesting of cells after incubation for subsequent analyses without cross-contamination (Fig. 1A). Although lacking direct cellular contact, this in vitro approach has been successfully applied for various applications including the characterization of insulin resistance of adipocytes as a result of the paracrine interaction of adipocytes with 
A

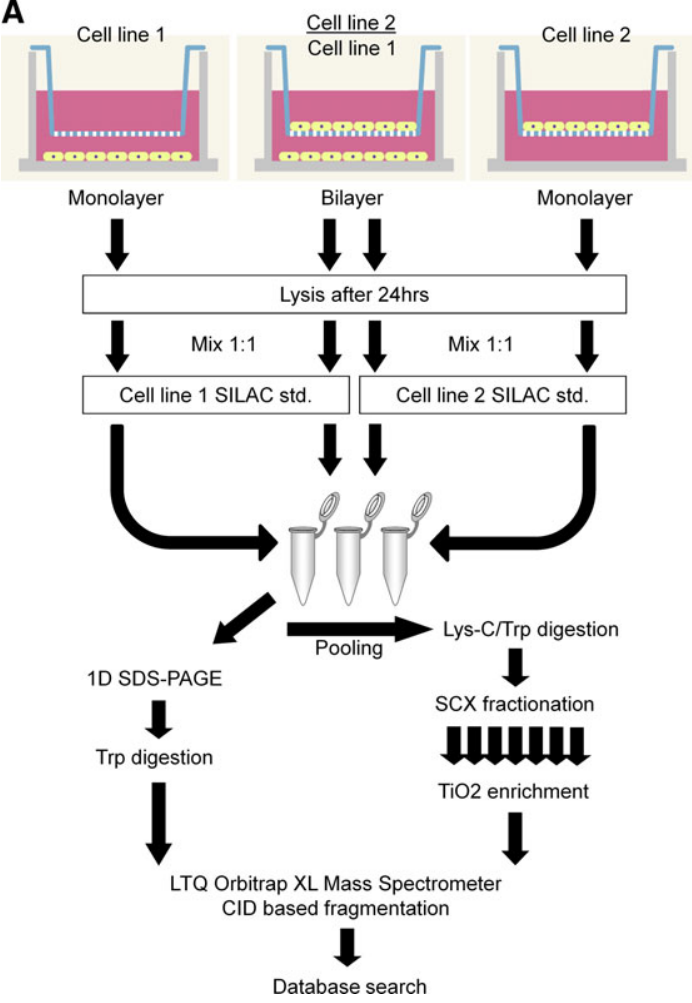

B
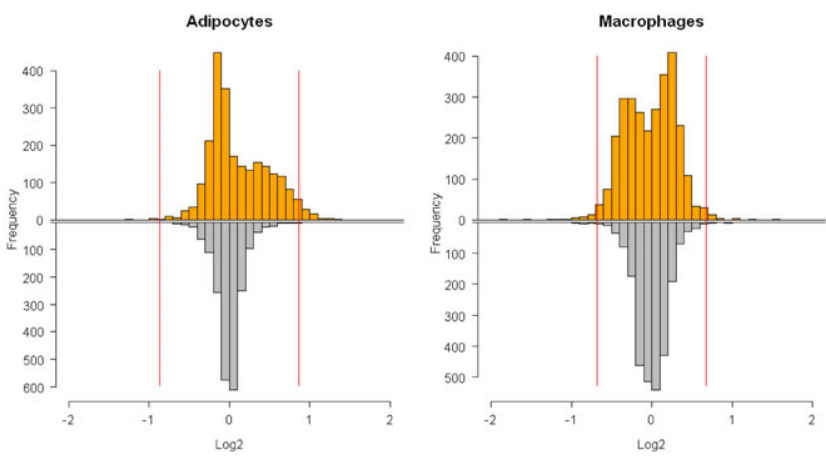

C

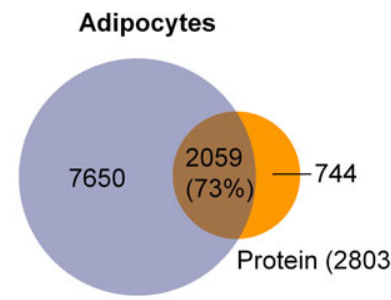

RNA (9709)

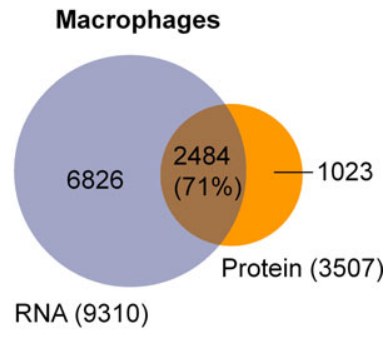

Figure 1. (A) Workflow comprising the cell cultivation on the bilayer system for $24 \mathrm{~h}$, harvest, lysis, addition of SILAC standard, prefractionation/digestion/enrichment for protein expression and phosphoprotein expression followed by MS measurement and database search. (B) Protein expression histogram for differentiated adipocytes (3T3-L1) and macrophages (RAW 264.7). Distribution in gray shows the log2 of the monolayer duplicates while the distribution displayed in orange shows the log2 bilayer versus monolayer expression. Red lines indicate the $1 \%$ cut-off limits for regulated proteins and were calculated 0.868 (1.8-fold) for adipocytes and 0.685 (1.6-fold) for macrophages. (C) Venn diagram of the overlap between detected mRNAs and identified proteins of adipocytes and macrophages.

macrophages $[4,6,10-13]$. In contrast to coculturing and subsequent sorting procedures such as fluorescence-activated cell sorting [14], the rapid harvest and processing using the transwell system preserves the molecules of the cells from potential degradation.

To grasp the pleiotropic effects of the interaction of adipocytes and macrophages on the protein level, we globally investigated the interactions between adipocytes and macrophages with regard to protein expression and phosphorylation changes by quantitative MS on a proteome-wide scale in this transwell system. To mimic low-grade inflammation in adipose tissue, we incubated differentiated murine 3T3-L1 adipocytes in the presence of murine macrophage RAW 264.7 cells in physiologically relevant ratios for $24 \mathrm{~h}$ without applying any additional artificial stimulus. Visual inspection of murine adipocytes exposed to macrophages did not reveal any striking morphological changes (Supporting Information Fig. 1). After $24 \mathrm{~h}$ cell-specific gene expression (increased levels of monocyte chemoattractant protein 1 of inflammation markers (Ccl2) for adipocytes [4] and increased levels of macrophage-inducible C-type lectin (Clec4e) for macrophages [10]) was in agreement with previous studies using the transwell system for adipocytes and macrophages
(Supporting Information Fig. 2), whereas earlier time points did not show striking upregulation of the markers $\mathrm{Ccl} 2$ and Clec4e. Later time points could not be analyzed due to complete consumption of media and strongly affected monocultured RAW 264.7 cells.

After harvesting, each cell line was mixed with the appropriate isotopically labeled cell line $[15,16]$, further processed, and detected quantitatively by using established stringent MS procedures (Supporting Information Material and Methods). For all MS analyses, we used biological duplicates, which were each processed twice (two technical replicates per biological sample). Further upscaling of the number of replicates would be desirable but is currently limited due to technical reasons. The biological duplicates showed very good correlations as determined by linear regression analyses (Supporting Information Fig. 3).

We then compared the protein expression patterns in adipocytes in the presence or absence of macrophages and also investigated the change of macrophage proteomes accordingly. Using conventional SDS-PAGE fractionation, we identified 4486 proteins in total with at least two unique peptides, 3243 proteins in 3T3-L1 adipocytes, and 3891 proteins in RAW 264.7 macrophages (Supporting Information Excel 
File 1). Fifty-nine percent of the proteins were detected in both cell lines (Supporting Information Fig. 4). This dataset was reduced to proteins with a designated SILAC ratio in bilayer and in monolayer samples, resulting in 2392 proteins specific for 3T3-L1 adipocytes and 2957 proteins for RAW 264.7 macrophages. The number of identified proteins using our approach was about one to two orders of magnitude higher than applying 2DE for quantification and MALDI-TOF MS [17], or alternative ESI-MS based methods for protein identification [18].

As shown in Fig. 1B the cut-off limits for up- and downregulation of proteins were chosen to include $99 \%$ of SILAC proteins $(1 \%$ would be false-positively regulated, further description can be found in Supporting Information Material and Methods). Applying these stringent criteria, we observed 73 strikingly upregulated and 14 strongly downregulated proteins ( $>1.8$-fold) in bilayer adipocytes, whereas macrophages exposed to differentiated adipocytes showed 27 strongly upregulated and 40 strikingly downregulated proteins (>1.6-fold, details can be found in Supporting Information Excel File 2).

More sensitive RNA microarray analyses showed several hundreds of regulated genes in adipocytes and macrophages (Supporting Information Excel File 3). More than 70\% of the identified proteins were also detected by RNA microarrays. But the transcripts of about up to $30 \%$ of detected proteins could not be found (Fig. 1C), mainly due to protein isoforms that do not exist on the RNA level and because of potentially different expression dynamics and stability of RNA and protein molecules. Notably, using functional-annotation enrichment analyses, these proteins were strikingly linked to acetylation (false discovery rate (FDR) $<\mathrm{E}-61$ ) and not to ubiquitin-like conjugation (false discovery rate $>\mathrm{E}+1$ ), indicating increased protein stability (Supporting Information Fig. 5). Since microarray-based RNA analysis includes molecule amplification, the detection sensitivity was in general higher than for protein analysis (approximately, threefold more transcripts were detected than proteins); however, improved MS detection devices may close this sensitivity gap in the future.

The rather high number of observed slightly regulated proteins is not uncommon in biology. Moreover, this observation reflects well the expected cellular state of low-grade inflammation induced by adipocyte-macrophage interaction [1]. To address the fundamental phenomenon of minor expression changes of interconnected mRNA and protein biomolecules to maintain cellular homeostasis, we applied protein and gene set enrichment analysis (PSEA and GSEA) as described recently [19]. Furthermore, the application of PSEA makes the biological data analysis robust against potential inaccuracies of detected expression fold changes of individual proteins or genes. The correlation of individual protein and mRNA expression was rather low (48\% for adipocytes and $64 \%$ for macrophages), probably due to various molecule dynamics and potentially experimental variation. However, analyses on the biological pathway levels clearly showed correlation (69\% for adipocytes, $73 \%$ for macrophages; Fig. 2A).

PSEA analyses of adipocytes and macrophages revealed a downregulation of energy-producing pathways such as oxidative phosphorylation and fatty acid oxidation, whereas upregulated pathways included cellular stress, cancer, and inflammation (Fig. 2B; protein-expression datasets used for PSEA can be found in Supporting Information Excel Files 4 and 5). Although we did not artificially boost strong inflammation response by using large or even unphysiological amounts of triggers such as lipopolysaccharides, the extracted pathways corroborated well the known underlying processes of the homeostasis of adipocytes due to paracrine interaction with macrophages $[1,4]$.

We further analyzed the phosphoproteome of the adipocyte-macrophage bilayer model using phosphopeptide enrichment protocols $[20,21]$. Altogether we detected over 12000 phosphorylation sites, $60 \%$ in 3T3-L1 adipocytes and $74 \%$ in RAW 264.7 macrophages (details can be found in Supporting Information Excel File 6). Interestingly, the overlap between identified phosphosites in adipocytes and macrophages was only 34\% (Supporting Information Fig. 6), suggesting cell line specific phosphorylation patterns. The phosphoproteome datasets were further reduced to allow only phosphopeptides containing one phosphosite per peptide and a SILAC ratio detected in both bilayer and monolayer experiments, resulting in 3200 phosphopeptides for adipocytes and 4514 phosphopeptides for macrophages (Supporting Information Excel File 6, 6A and 6B). Using our analysis criteria and cut-off limits (Supporting Information Material and Methods), 83 phosphosites were found to be strongly upregulated and 24 phosphosites were strikingly downregulated for differentiated adipocytes that were exposed to the macrophages. For macrophages that were exposed to adipocytes, 70 phosphosites were found to be strongly upregulated and 63 were found to be downregulated (Supporting Information Excel File 7).

We further investigated the potentially involved signaling networks, and focused on the analysis of enriched kinase activities in the large phosphoproteome datasets using prior knowledge of kinase-substrates interactions as described in Supporting Information Material and Methods. Thereby we obtained a comprehensive, unbiased, and quantitative overview of activated and inactivated kinases based on their observed hyper- and hypophosphorylated target sites (Fig. 2C). These phosphoproteome data confirmed well previous biological and physiological studies [1]. For example, the interaction of adipocytes and macrophages resulted in strong activation of the kinase AKT1, which in general leads to inhibiting apoptosis and increasing protein synthesis. Observed activated kinases including RSK2 or PKCZ are also known to be involved in downregulating apoptosis. RSK2, a downstream target of ERK1/2, phosphorylates p90RSK, a stress response factor, which further inhibits apoptosis, whereas PKCZ activation stimulates inflammatory response. Further, the activation of CDK1 and PAK1, in particular 
A

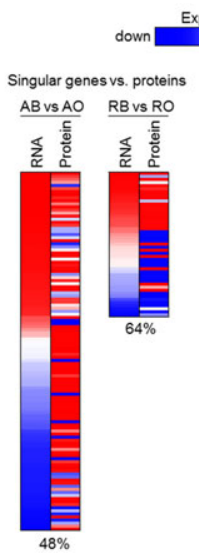

Pathways (KEGG)

ABvsAO RBvsRO

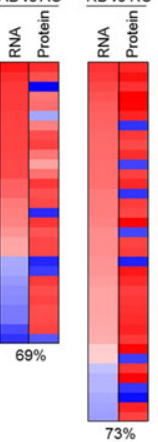

C

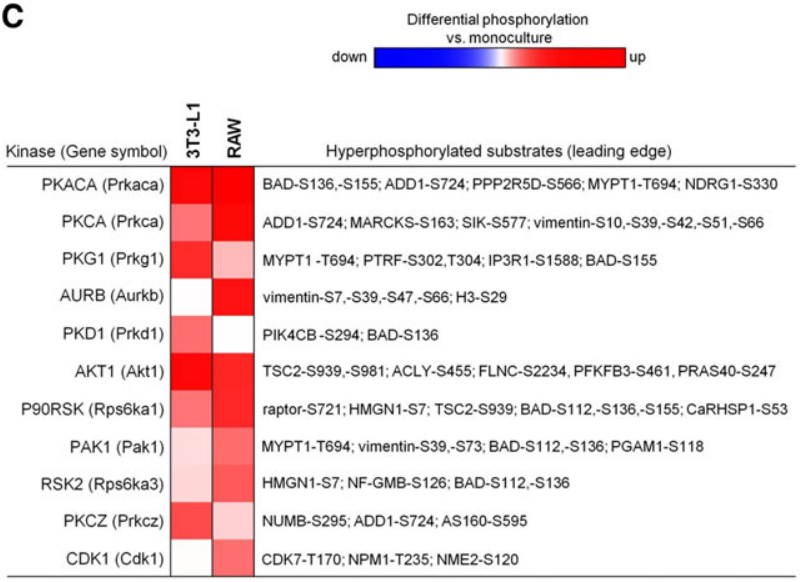

B

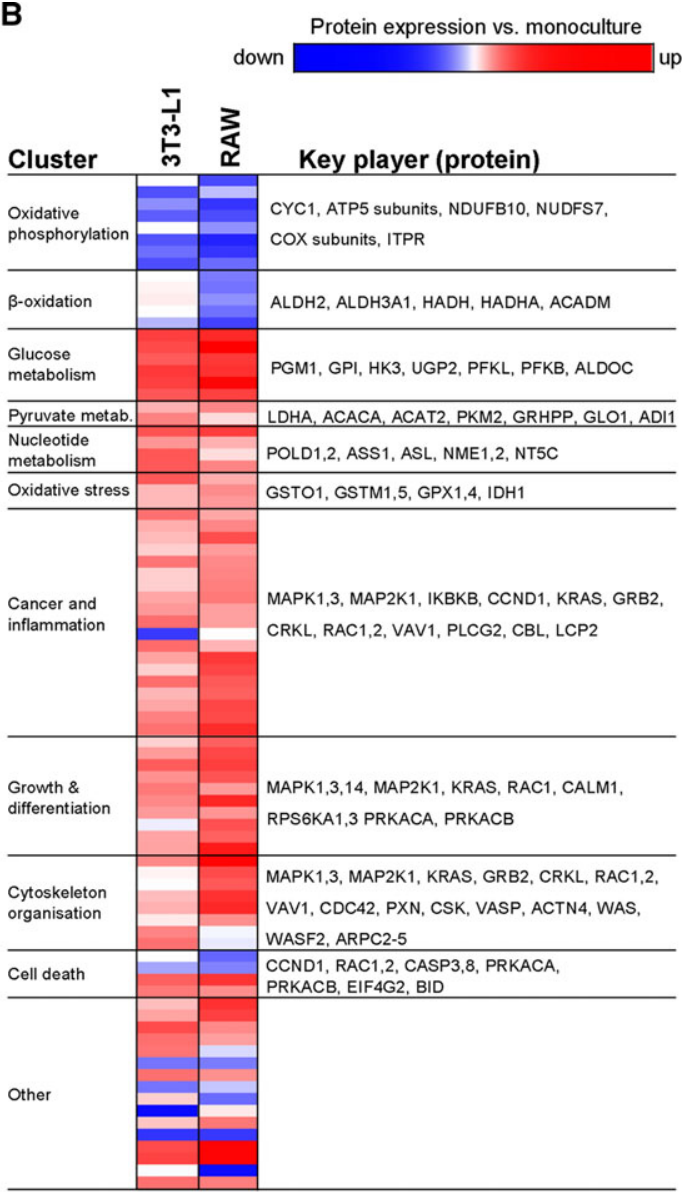

Figure 2. (A) Comparison of transcriptome and proteome on the single gene level (left) and on the pathway level (right). (B) Heatmap of protein set enrichment analysis in adipocytes and macrophages. Red boxes indicate upregulation and boxes in blue indicate downregulation of kyoto encyclopedia of genes and genomes (KEGG) pathways in the bilayer system. Pathways are clustered according to their overlap in key player proteins and labeled with the most common function of this cluster. (C) Heatmap for adipocytes and macrophages displaying the intensity of substrate phosphosites for specific kinase proteins. Red boxes indicate upregulation of kinase activity in the bilayer system, based on overrepresentation of known phosphosite substrates for that kinase (indicated in the right panel).

in the macrophage, indicates proliferation events including the regulation of the cytoskeleton and cell motility. Most strikingly, the activation of the kinases PKCA and PKCZ in the adipocytes and the macrophages can lead to negative regulation of insulin receptor signaling, which may explain in part on the cellular level the development of insulin resistance due to macrophage infiltration in adipose tissue. These data support the use of anti-inflammatory molecules such as salicylates or amorfrutins to counteract kinase signaling cascades to eventually increase insulin sensitivity $[7,22,23]$.

We found, among others, modified kinase substrates involved in cell progression and proliferation such as CDK7; specific phosphorylation of the cell death promoting protein BAD, which is associated with decreased apoptosis; and a number of proteins involved in cellular organization and structure such as vimentin, ADD1, MYPT1, HMGN1, or NUMB (details can be found in Fig. 2C). However, the role of some of these substrates is in part still largely unknown, in particular in the context of adipocytes or macrophages. Further, the observed AKT1-dependent phosphorylation of TSC2 inhibits cellular apoptosis, whereas the detected phosphorylation of raptor influences cell growth via the mTORC1 pathway.

In general, the phosphosite datasets are in line with the observed protein expression data. For example, the increased protein expression of "cancer and inflammation" and "growth and differentiation" pathways can be easily brought in context with the activation of the described kinase signaling events (Fig. 2B and C).

In summary, the holistic analysis of the described proteome-wide expression and phosphorylation datasets of adipocytes and macrophages interacting in vitro revealed a subtle interplay of cellular pathways including a number of known and so far less studied proteins and molecular pathways, which could only be difficult to decipher by focusing on only few markers. We could largely confirm 
the protein-based data by genome-wide mRNA expression analysis. The generated protein datasets are freely available (ftp://PASS00203:ES8494hq@ftp.peptideatlas.org). These data provide an unbiased view on the paracrine interaction of adipocytes and macrophages and a resource to generate new hypotheses for further experiments including the dynamics of the interaction of the cells, drug treatments, or other interventions. For example, various bacterial lipopolysaccharides or toxines can be used to dissect with the transwell system the potentially causative role of bacteria in inflammation processes leading to insulin resistance in obese subjects [24]. In gereral the applied quantitative proteomics cell bilayer (qPCB) in vitro method can be used for various cell-cell combinations to shed light on the role of local biological microenvironments on pathophysiology.

Our work is supported by the German Ministry for Education and Research (BMBF, grant 0315082 (01EA1303) to S.S.), the European Union (FP7/2007-2013, under grant agreement 262055 (ESGI) to S.S.), and the Max Planck Society.

The authors have declared no conflict of interest.

\section{References}

[1] Gregor, M. F., Hotamisligil, G. S., Inflammatory mechanisms in obesity. Annu. Rev. Immunol. 2011, 29, 415-445.

[2] Xu, H., Barnes, G. T., Yang, Q., Tan, G. et al., Chronic inflammation in fat plays a crucial role in the development of obesity-related insulin resistance. J. Clin. Invest. 2003, 112, 1821-1830.

[3] Pal, D., Dasgupta, S., Kundu, R., Maitra, S. et al., Fetuin-A acts as an endogenous ligand of TLR4 to promote lipid-induced insulin resistance. Nat. Med. 2012, 18, 1279-1285.

[4] Lumeng, C. N., Deyoung, S. M., Saltiel, A. R., Macrophages block insulin action in adipocytes by altering expression of signaling and glucose transport proteins. Am. J. Physiol. 2007, 292, E166-E174.

[5] Permana, P. A., Menge, C., Reaven, P. D., Macrophagesecreted factors induce adipocyte inflammation and insulin resistance. Biochem. Biophys. Res. Commun. 2006, 341, 507-514.

[6] Keuper, M., Bluher, M., Schon, M. R., Moller, P. et al., An inflammatory micro-environment promotes human adipocyte apoptosis. Mol. Cell Endocrinol. 2011, 339, 105-113.

[7] Yuan, M., Konstantopoulos, N., Lee, J., Hansen, L. et al., Reversal of obesity- and diet-induced insulin resistance with salicylates or targeted disruption of Ikkbeta. Science 2001, 293, 1673-1677.

[8] Ong, S. E., Mann, M., A practical recipe for stable isotope labeling by amino acids in cell culture (SILAC). Nat. Protoc. 2006, 1, 2650-2660.

[9] Bassols, J., Ortega, F. J., Moreno-Navarrete, J. M., Peral, B. et al., Study of the proinflammatory role of human differentiated omental adipocytes. J. Cell Biochem. 2009, 107, 1107-1117.
[10] Ichioka, M., Suganami, T., Tsuda, N., Shirakawa, I. et al., Increased expression of macrophage-inducible C-type lectin in adipose tissue of obese mice and humans. Diabetes 2011, $60,819-826$.

[11] Reynolds, C. M., McGillicuddy, F. C., Harford, K. A., Finucane, O. M. et al., Dietary saturated fatty acids prime the NLRP3 inflammasome via TLR4 in dendritic cellsimplications for diet-induced insulin resistance. Mol. Nutr. Food Res. 2012, 56, 1212-1222.

[12] McGillicuddy, F. C., Harford, K. A., Reynolds, C. M., Oliver, E. et al., Lack of interleukin-1 receptor I (IL-1RI) protects mice from high-fat diet-induced adipose tissue inflammation coincident with improved glucose homeostasis. Diabetes 2011, 60, 1688-1698.

[13] Samuvel, D. J., Jin, J., Sundararaj, K. P., Li, Y. et al., TLR4 activation and IL-6-mediated cross talk between adipocytes and mononuclear cells synergistically stimulate MMP-1 expression. Endocrinology 2011, 152, 4662-4671.

[14] Moody, M. A., Haynes, B. F., Antigen-specific B cell detection reagents: use and quality control. Cytometry $A$ 2008, 73, 1086-1092.

[15] Monetti, M., Nagaraj, N., Sharma, K., Mann, M., Large-scale phosphosite quantification in tissues by a spike-in SILAC method. Nat. Methods 2011, 8, 655-658.

[16] Bendall, S. C., Hughes, C., Stewart, M. H., Doble, B. et al., Prevention of amino acid conversion in SILAC experiments with embryonic stem cells. Mol. Cell Proteomics 2008, 7, 1587-1597.

[17] Dvorzhinski, D., Thalasila, A., Thomas, P. E., Nelson, D. et al., A novel proteomic coculture model of prostate cancer cell growth. Proteomics 2004, 4, 3268-3275.

[18] Li, M., Aliotta, J. M., Asara, J. M., Wu, Q. et al., Intercellular transfer of proteins as identified by stable isotope labeling of amino acids in cell culture. J. Biol. Chem. 2010, 285, 6285-6297.

[19] Meierhofer, D., Weidner, C., Hartmann, L., Mayr, J. A. et al., Protein sets define disease states and predict in vivo effects of drug treatment. Mol. Cell Proteomics 2013, 12, 1965-1979.

[20] Wisniewski, J. R., Zougman, A., Mann, M., Combination of FASP and StageTip-based fractionation allows in-depth analysis of the hippocampal membrane proteome. J. Proteome Res. 2009, 8, 5674-5678.

[21] Villen, J., Gygi, S. P., The SCX/IMAC enrichment approach for global phosphorylation analysis by mass spectrometry. Nat. Protoc. 2008, 3, 1630-1638.

[22] Weidner, C., de Groot, J. C., Prasad, A., Freiwald, A. et al., Amorfrutins are potent antidiabetic dietary natural products. Proc. Natl. Acad. Sci. US A 2012, 109, 7257-7262.

[23] Weidner, C., Wowro, S. J., Freiwald, A., Kawamoto, K. et al., Amorfrutin $B$ is an efficient natural peroxisome proliferatoractivated receptor gamma (PPARgamma) agonist with potent glucose-lowering properties. Diabetologia 2013, 56, 1802-1812.

[24] Zhao, L., The gut microbiota and obesity: from correlation to causality. Nat. Rev. Microbiol. 2013, 11, 639-647. 